\title{
Gastritis Associated with Epstein-Barr Virus Infection
}

\author{
Akari Hisamatsu ${ }^{1}$, Takayuki Nagai ${ }^{1}$, Hitoshi Okawara ${ }^{1}$, Hiroshi Nakashima ${ }^{1}$, Takako Tasaki ${ }^{1}$, \\ Yoshifumi Nakagawa ${ }^{1}$, Masahiko Hashinaga ${ }^{1}$, Shunichi Kai ${ }^{2}$, Shigeo Yokoyama ${ }^{3}$, \\ Kazunari Murakami ${ }^{4}$ and Toshio Fujioka ${ }^{4}$
}

\begin{abstract}
Infectious mononucleosis is a self-limiting clinical syndrome caused by primary Epstein-Barr virus (EBV) infection. EBV-associated gastritis, however, has rarely been documented. We report a case of a 17-year-old woman who presented with fever, sore throat, and epigastric pain. Upper endoscopy revealed diffuse granular mucosae and elevated lesions in the stomach. Histologically, the biopsied mucosa was infiltrated by numerous atypical lymphocytes. From clinical, histopathologic, immunohistochemical, and in situ hybridization analyses, we diagnosed EBV-associated gastritis. Her symptoms spontaneously resolved, and follow-up endoscopy revealed improvement and no atypical lymphocytes. To prevent misdiagnosis and unnecessary treatment, the possibility of EBV-associated gastritis should be considered.
\end{abstract}

Key words: Epstein-Barr virus, infectious mononucleosis, gastritis, malignant lymphoma, endoscopy

(Inter Med 49: 2101-2105, 2010)

(DOI: 10.2169/internalmedicine.49.3789)

\section{Introduction}

Infectious mononucleosis (IM) is caused by primary Epstein-Barr virus (EBV) infections, and typical manifestations of IM include fever, malaise, pharyngitis, lymphadenopathy, and hepatic involvement. The association of EBV with malignant lymphoma (1) and carcinoma $(2,3)$ of the stomach is well documented, but gastrointestinal lesions have rarely been observed as a complication of IM. EBV gastritis is rarely recognized, and to our knowledge, only 4 cases have previously been reported (4-7). Here, we present a fifth case of EBV gastritis, in which the gastritis was associated with lymphocytic infiltration and it was difficult to differentiate from malignant lymphoma by histopathological analyses.

\section{Case Report}

A 17-year-old woman presented with a 6-day history of intermittent fever, fatigue, sore throat, headache, nausea, and epigastric pain. She had no symptom of vomiting, diarrhea, weight loss, or cough. There was no similar presentation among her family members.

Physical examination revealed acute distress with a body temperature of $37.1^{\circ} \mathrm{C}$. There was no skin rash or jaundice. Peripheral lymphadenopathy of the neck and tonsillitis were noted. Her abdomen was soft with normal bowel sounds, and diffuse tenderness was observed in the upper quadrants. No obvious organomegaly was detected on palpation. The patient had no significant medical history and was not taking aspirin or nonsteroidal anti-inflammatory drugs.

Laboratory tests showed an increased white blood cell count $(9,440 / \mu \mathrm{L})$, with $22.0 \%$ atypical lymphocytes in the peripheral blood. Her hemoglobin level was $12.1 \mathrm{~g} / \mathrm{dL}$, hematocrit, 39.2\%, and platelet count, $123,000 / \mu \mathrm{L}$. Her liver function test results were abnormal, including elevations in alkaline phosphatase (515 IU/L), aspartate transaminase (110 IU/L), alanine transaminase (222 IU/L), serum lactate dehydrogenase (454 IU/L), and $\gamma$-GTP (397 IU/L). All other biochemical tests were unremarkable. Immuno-serologic tests for hepatitis B viral surface antigen and antibodies against

\footnotetext{
${ }^{1}$ Department of Gastroenterology, Oita Kouseiren Tsurumi Hospital, Beppu, ${ }^{2}$ Department of Diagnostic Pathology, Oita Kouseiren Tsurumi Hospital, Beppu, ${ }^{3}$ Department of Diagnostic Pathology, Oita University, Faculty of Medicine, Yufu and ${ }^{4}$ Department of General Medicine and Gastroenterology, Oita University, Faculty of Medicine, Yufu

Received for publication April 10, 2010; Accepted for publication June 16, 2010

Correspondence to Dr. Akari Hisamatsu, akari.m@med.oita-u.ac.jp
} 

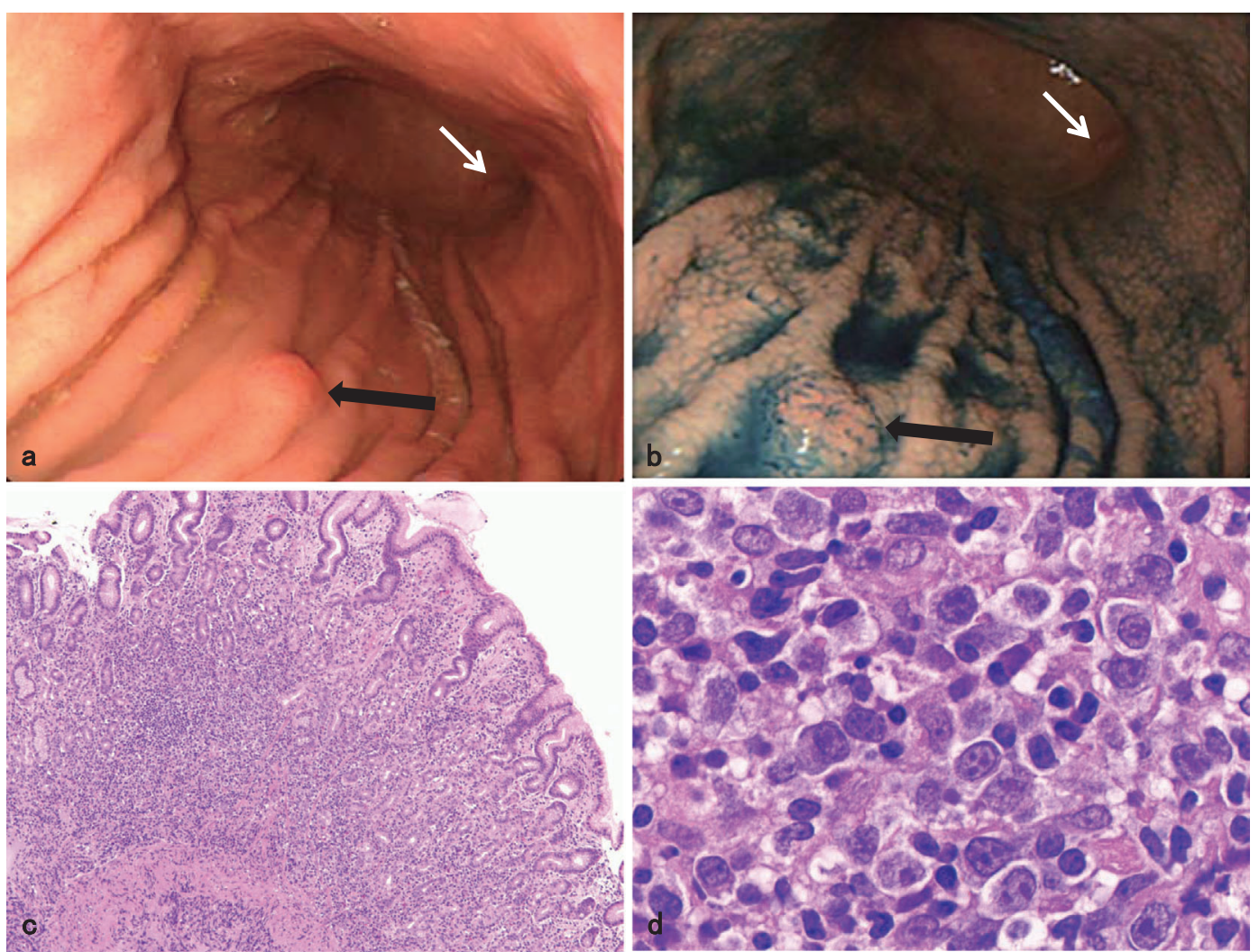

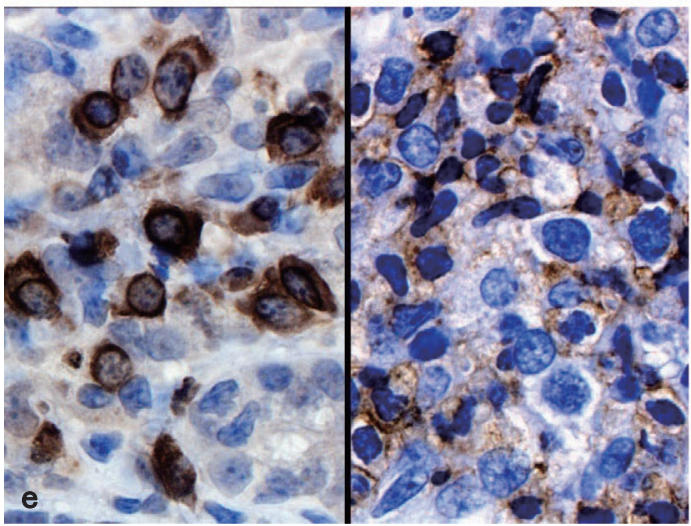

CD79a

CD45RO

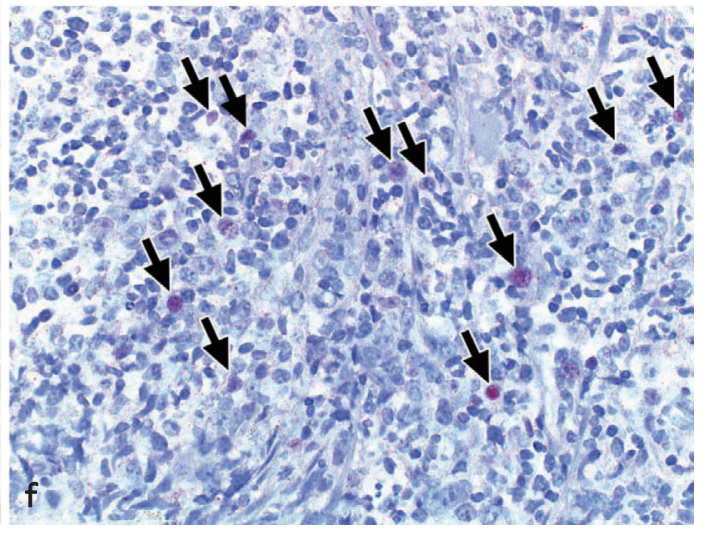

EBER

Figure 1. Characteristics of the patient at her first examination. a) Endoscopic image showing granular mucosa with several elevated lesions (arrows). b) Narrow band image (NBI) clearly showing diffuse granular mucosae. c) Histological findings of the biopsy specimen, showing a dense and diffuse lymphocytic infiltrate in the lamina propria (Hematoxylin and Eosin staining, original magnification $\times 100$ ). d) High-power view showing a mixture of lymphocytes, immunoblasts, and plasma cells (Hematoxylin and Eosin staining, original magnification $\times 1000$ ). e) Immunohistochemical labeling for CD79a and CD45RO (original magnification $\times 1000$ ). f) In situ hybridization results showing numerous EBER-positive lymphocytes (arrows) in the lamina propria (original magnification $\times 400)$.

hepatitis $\mathrm{C}$ virus and cytomegalovirus were negative. The most significant laboratory findings were elevated levels of serum EBV antibodies, including IgG viral capsid antigen (VCA; 160), IgM VCA (40), IgG early antigen (EA; 10), and Epstein-Barr nuclear antigen (EBNA; <10).

The patient underwent a neck and abdominal ultrasound (US), which showed multiple enlarged lymph nodes in her neck, hepatosplenomegaly, thickened gallbladder wall, and hepatoduodenal lymphadenopathy. Upper endoscopy re- vealed diffuse granular mucosae and several elevated lesions in the gastric body and angle without ulceration (Fig. 1a, 1b). The antrum and pylorus were less inflamed. Endoscopy revealed that the esophageal and duodenal mucosae were intact.

One gastric biopsy was taken from each site of the elevated lesion and granular mucosa, and microscopic examination of Hematoxylin and Eosin (H\&E)-stained slides of the former, but not the latter, showed a marked expansion of the 

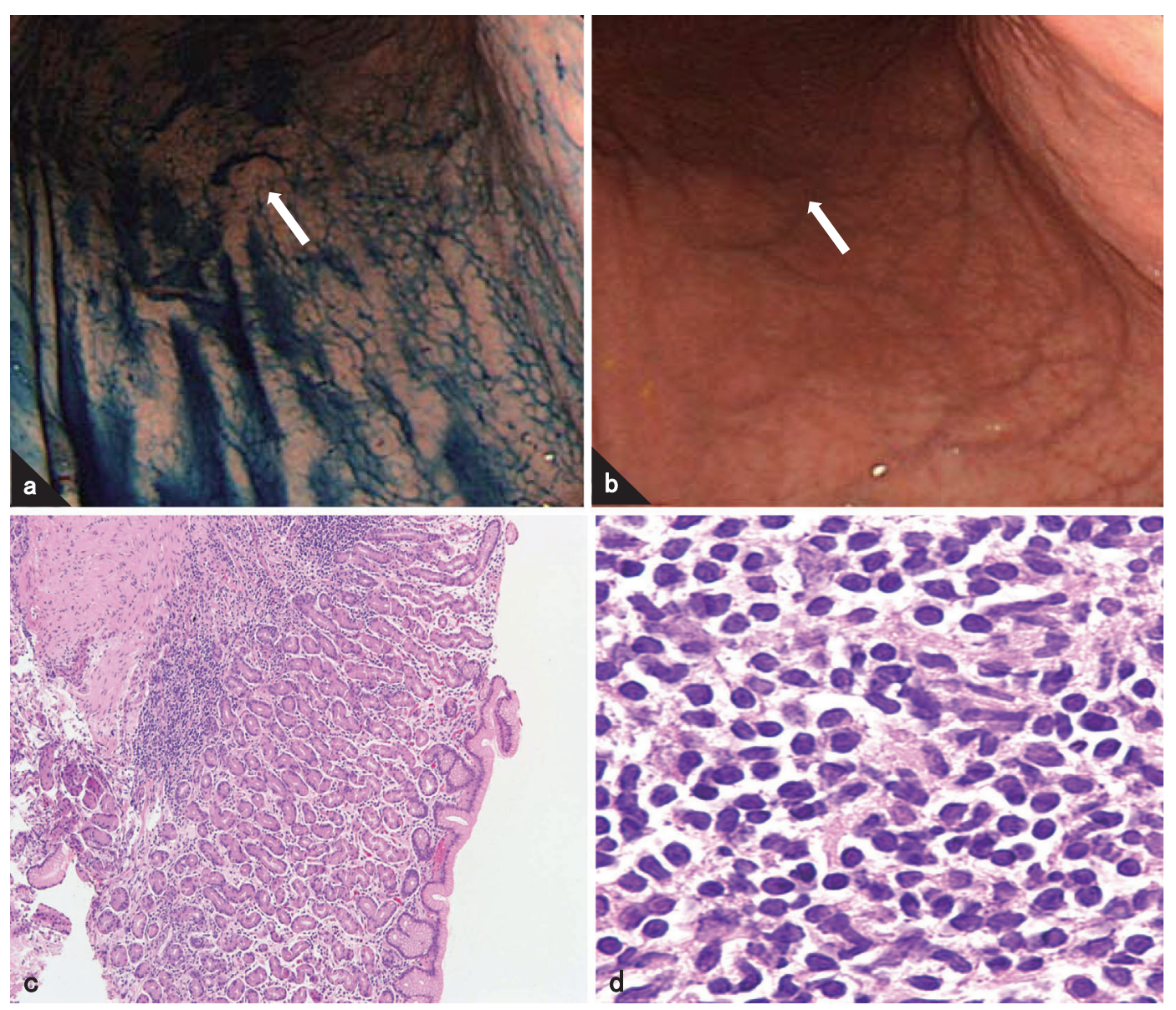

Figure 2. Characteristics of the patient after 6 weeks. a) Endoscopic image showing improvement in elevated lesions but continued presence of granular mucosa (arrow). b) NBI clearly showing the remaining granular mucosae. c) Histopathologic findings of the gastric mucosa biopsy specimen, showing only slight lymphoid infiltration (Hematoxylin and Eosin staining, original magnification $\times 100)$. d) High-power view of the image in $2 \mathrm{c}$ (Hematoxylin and Eosin staining, original magnification $\times \mathbf{8 0 0})$.

lamina propria by a dense, diffuse, and predominantly lymphoid infiltrate (Fig. 1c). Despite the presence of surface erosion, neutrophils were not prominent. The epithelial mucosae showed reactive change without distinct atypia. The lymphoid infiltrate was polymorphic, consisting of small, intermediate, and large lymphocytes (Fig. 1d), composed of a mixture of B lymphocytes with CD79a and T lymphocytes with CD45RO (Fig. 1e). Helicobacter pylori (H. pylori) was not identified, and the absence of neoplastic glands ruled out the possibility of gastric adenocarcinoma. On the basis of these results, our pathologist suspected malignant lymphoma, although the patient's clinical characteristics led him to perform an immunohistochemical assay to test for EBV. In situ hybridization confirmed the presence of atypical lymphocytes positive for EBV (Fig. 1f). Thus, we concluded that the gastritis was a manifestation of IM.

The patient was treated with supportive care on an out patient basis. Fever and pharyngitis disappeared within 10-12 days. Peripheral lymphadenopathy of the neck persisted for several more weeks. The gastrointestinal symptoms subsided within 8 days. An endoscopy performed 6 weeks after diag- nosis, revealed improvements in the elevated lesions but persistence of the granular mucosae (Fig. 2a, 2b). Biopsies were taken from both lesions, and only slight lymphoid infiltration was identified from all specimens (Fig. 2c, 2d). These specimens were also subjected to in situ hybridization analyses, which indicated that none of the lymphocytes were EBV-positive. The patient's liver function tests and white blood cell count were also completely normalized, and the patient has remained healthy, with no gastrointestinal complaints, for 10 months.

\section{Discussion}

This report describes a case of acute EBV gastritis as part of an IM manifestation. We attempted to find descriptions of similar cases in the literature, but a MEDLINE search of all English-language sources from 1983 to 2010 revealed only 4 previous reports of this condition (4-7). The present patient had similar clinicopathologic features to these previously reported occurrences, and those patients, like our patient, received only symptomatic management (Table 1). 
Table 1. EBV Gastritis of the Past English Literature

\begin{tabular}{|c|c|c|c|c|c|c|c|}
\hline & Author & Year & $\begin{array}{l}\text { Age } \\
\text { (years) }\end{array}$ & Sex & $\begin{array}{l}\text { Medical } \\
\text { history }\end{array}$ & Chief complaint & Endoscopic findings \\
\hline 1 & Kitayama Y & 2000 & 40 & M & None & fever, diarrhea & multiple small irregular ulcers \\
\hline 2 & Zhang Y & 2003 & 58 & unknown & $\begin{array}{l}\text { type } 1 \\
\text { diabetes }\end{array}$ & $\begin{array}{l}\text { abdominal pain, anorexia, } \\
\text { vomiting, diarrhea }\end{array}$ & $\begin{array}{l}\text { thickened, edematous and inflamed } \\
\text { rugal folds without ulcer }\end{array}$ \\
\hline 3 & Zong-ming $\mathrm{E}$ & 2007 & 59 & M & $\begin{array}{l}\text { asthma, } \\
\text { hypertension }\end{array}$ & epigastric pain, fever, nausea & $\begin{array}{l}\text { diffusely granular and erythematous, } \\
\text { mucosa and numerous ulcers }\end{array}$ \\
\hline 4 & Scott R & 2008 & 18 & $\mathrm{~F}$ & none & epigastric pain, fever, vomiting & nodular mucosa and a solitary ulcer \\
\hline 5 & Present case & 2009 & 17 & $\mathrm{~F}$ & none & $\begin{array}{l}\text { epigastric pain, fever, sore } \\
\text { throat }\end{array}$ & granular mucosa and erosion \\
\hline
\end{tabular}

In the historical cases, endoscopy revealed several ulcerative lesions and diffuse gastritis (4-7). The distal portion of the body and the antrum tended to exhibit less inflammation (5), while the esophageal and duodenal mucosae were intact in all cases (4-7). The present case revealed diffuse granular mucosae and several elevated lesions in the gastric body and the angle without ulceration. Although the appearance of the granular mucosa suggested Helicobacter gastritis, no $H$. pylori was detected, and the urine Helicobacter antibody test was negative, indicating that this symptom was the result of a nonspecific immune response.

The 4 previous reports of EBV-associated gastritis indicated that the cardinal histopathological sign of the condition is a dense and diffuse lymphoid infiltrate with atypical lymphocytes (4-7). Indeed, these symptoms were observed in the present patient. Initially, our pathologist originally suspected malignant lymphoma of the stomach, rather than hyperplasia or mucosa-associated lymphoid tissue (MALT) and its malignant phenotype, because of the high grade of atypia. However, in this case, the pathologist conducted an in situ hybridization to search for EBV RNA in the biopsy specimens because the patient's detailed clinical information suggested that EBV, rather than malignant lymphoma, might be the cause of her symptoms. This highlights the importance of using both clinical and pathological data before attempting a diagnosis.

As mentioned in previous case reports (4-7), in situ hybridization techniques are very useful in diagnosing EBVassociated gastritis. However, since most of the population has a latent infection, the mere presence of EBV in tissues is insufficient evidence for its etiologic role in the development of gastric symptoms. Furthermore, the detection of EBV does not, by itself, support the assertion that the gastric symptoms are benign (5). A wide variety of neoplasms, including malignant lymphomas and gastric carcinomas, have been associated with EBV (8). Therefore, it is important to consider clinical, serological, and histological find- ings as well as the in situ hybridization results when determining whether the illness is caused by a primary response to EBV infection.

In conclusion, we encountered a rare case of EBVassociated gastritis that histologically simulated gastric lymphoma. In many cases, this condition might be overlooked, since physicians do not routinely conduct endoscopic examinations for common viral syndromes, particularly in young patients such as the present case (7). Our case shows that it is vital for clinicians to be aware that EBV-associated gastritis does occur, although perhaps only rarely, and indicates how important it is for clinicians and pathologists to share information when determining a diagnosis. In the future, the correct diagnosis of EBV-associated gastritis could avoid unnecessary invasive treatment.

\section{References}

1. Lee SS, Jang JJ, Cho KJ, Khang SK, Kim CW. Epstein-Barr virus associated primary gastrointestinal lymphoma in nonimmunocompromised patient in Korea. Histopathology 30: 234242, 1997.

2. Sidagis J, Ueno K, Tokunaga M, Ohyama M, Eizuru Y. Molecular epidemiology of Epstein-Barr virus (EBV) in EBV-related malignancies. Int J Cancer 72: 72-76, 1997.

3. Greiner A, Kirchner T, Ott G, Marx A, Fischbach W. MullerHermelink HK. Occurrence of multiple lymphoepithelioma-like carcinoma and MALT-type lymphoma in the stomach: detection of EBV in carcinoma but not in lymphoma. Histopathology 29: 5156, 1996.

4. Kitayama Y, Honda S, Sugimura H. Epstein-Barr virus-related gastric pseudolymphoma in infectious mononucleosis. Gastrointest Endosc 52: 290-291, 2000.

5. Zhang Y, Molot R. Severe gastritis secondary to Epstein-Barr viral infection: unusual presentation of infectious mononucleosis and associated diffuse lymphoid hyperplasia in gastric mucosa. Arch Pathol Lab Med 127: 478-480, 2003.

6. Chen ZM, Shah R, Zuckerman GR, Wang HL. Epstein-Barr virus gastritis; an underrecognized form of severe gastritis simulating gastric lymphoma. Am J Surg Pathol 31: 1446-1449, 2007.

7. Owens SR, Walls A, Krasinskas A, Rund CR. Epstein-Barr virus 
Inter Med 49: 2101-2105, 2010 DOI: 10.2169/internalmedicine.49.3789

gastritis: rare or rarely sampled? A case report. Int J Surg Pathol May 21: 1-3, 2008.
8. Anagnostopoulos I, Hummel M. Epstein-Barr virus in tumors. Histopathology 29: 297-315, 1996.

(C) 2010 The Japanese Society of Internal Medicine http://www.naika.or.jp/imindex.html 